\title{
Constitutive overexpression of the pollen specific gene SKS13 in leaves reduces aphid performance on Arabidopsis thaliana
}

\author{
Xi Chen ${ }^{1}$, Zhao Zhang ${ }^{2}$, Richard G F Visser ${ }^{1}$, Ben Vosman ${ }^{1 *}$ and Colette Broekgaarden ${ }^{1}$
}

\begin{abstract}
Background: Plants have developed a variety of mechanisms to counteract aphid attacks. They activate their defences by changing the expression of specific genes. Previously we identified an activation tag mutant of Arabidopsis thaliana on which Myzus persicae population development was reduced. Activation tag mutants are gain-of-function in which the expression of a gene is increased by the insertion of the Cauliflower mosaic virus 355 enhancer that acts on the natural promoter. By further characterizing this previously identified mutant we identified a gene that reduces performance of $M$. persicae and also provided clues about the mechanism involved.

Results: We show that SKU5 SIMILAR 13 (SKS13), a gene whose expression in wild type plants is restricted to pollen and non-responsive to $M$. persicae attack, is overexpressed in the $A$. thaliana mutant showing reduced performance of M. persicae. Monitoring M. persicae feeding behaviour on SKS13 overexpressing plants indicated that M. persicae have difficulties feeding from the phloem. The constitutive expression of SKS13 results in accumulation of reactive oxygen species, which is possibly regulated through the jasmonic acid pathway. The enhanced resistance is not aphid species specific as also the population development of Brevicoryne brassicae was affected.

Conclusions: We demonstrate that constitutive expression in leaves of the pollen-specific gene SKS13 can enhance plant defence, resulting in a reduction of $M$. persicae population development and also decreases the transmission of persistent viruses. Overexpression of SKS13 in A. thaliana also affects B. brassicae and possibly other phloem feeding insects as well. Identifying genes that can enhance plant defence against insects will be important to open up new avenues for the development of insect resistant crop plants.
\end{abstract}

Keywords: Activation tag mutant, Brevicoryne brassicae, Electrical penetration graph, Jasmonic acid, Myzus persicae, Phloem-feeding insect, Reactive oxygen species

\section{Background}

Aphids have a sophisticated feeding strategy in which they use their stylets to penetrate plant tissue and puncture cells along the intercellular pathway towards the phloem [1]. To facilitate the probing and feeding processes, aphids secrete saliva into the plant tissue to degrade cell walls and to overcome occlusion of the feeding site $[2,3]$. Once an aphid establishes a feeding site it can feed from the phloem of a susceptible plant for hours or even days [1]. Aphid infestation limits plant productivity due to the depletion of photo-assimilates and the deposition of excess sugars

\footnotetext{
* Correspondence: ben.vosman@wur.nl

${ }^{1}$ Wageningen UR, Plant Breeding, PO. Box 386, Wageningen 6700 AJ, the Netherlands

Full list of author information is available at the end of the article
}

as honeydew that encourages growth of mold. In addition, aphids are important vectors of numerous plant viruses that can be transmitted during probing and feeding, resulting in additional damage to plants [4].

Plants have evolved a series of defense traits to directly affect the aphid's feeding behavior. These defenses include physical and chemical traits that can be constitutively present or induced upon aphid attack [5]. Physical traits, such as hairs and glandular trichomes, hinder aphid settling on a plant [6]. Chemical traits include the production of secondary metabolites and proteins that are repellent or toxic to aphids thereby affecting their performance [7]. For example, the brassicaceous-specific secondary metabolites glucosinolates have been shown to negatively affect the performance of the generalist 
aphid Myzus persicae [8]. Contrary to constitutive traits, inducible defenses require recognition of the attacking aphid and subsequent transcriptional reprogramming. This also includes the activation of general wound responses. An increasing body of evidence suggests that reactive oxygen species (ROS), which were always thought to be induced as a general wound response, can play a role in plant defense towards aphids as well $[9,10]$. For example, an early accumulation of ROS upon Russian wheat aphid infestation was suggested to be a defense response in aphid resistant wheat [11]. In contrast, an increasing concentration of ascorbic acid, a compound that is capable of reducing ROS, leads to an enhanced aphid fecundity [10], further underpinning the role of ROS in plant defense towards aphids. Moreover, ROS can act as signaling molecules, along with JA, to confer aphid resistance [12]. The activation of plant hormone pathways, especially jasmonic acid (JA), salicylic acid (SA) and ethylene (ET), plays an important role in plant defense against aphids $[13,14]$. These pathways interact in a network, regulating the expression of specific groups of defense-related genes [15]. Although all pathways can be involved in defense, the JA pathway is thought to be the most effective against aphids $[16,17]$. Constitutive activation of the JA pathway in an Arabidopsis thaliana mutant leads to enhanced aphid resistance, whereas blocking the JA pathway results in aphid susceptibility [14].

It has been shown that certain genes, for instance IQD1 (IQ-Domain1) and MPL1 (Myzus persicae -induced lipase 1) can confer plant resistance to insects when their level of expression is increased or the location of expression is changed [18-20]. Such genes may be identified by screening activation tag mutant collections for insect resistance [18,21]. In these mutants, tagged genes are overexpressed by a tetramer Cauliflower mosaic virus (CaMV) 35S enhancer adjacent to the natural promoter, resulting in a dominant gain-offunction phenotype [22]. By screening such a mutant collection of $A$. thaliana, we have identified several mutants with enhanced resistance against $M$. persicae [23]. In the present paper we characterize one of these mutants, leading to the identification of SKUS SIMILAR 13 (SKS13) as a gene responsible for enhanced resistance to $M$. persicae. We analyzed the feeding behavior of $M$. persicae on the mutant using the electrical penetration graph (EPG) technique [24] to get information about the location of resistance factors. Based on the putative involvement of SKS13 in oxidation/reduction reactions we visualized the accumulation of ROS in leaves. Finally, we monitored the expression of several JA-, SA, and ethylene-pathway marker genes to study the possible interaction of SKS13 with these hormone pathways that may explain the aphid resistance conferred by SKS13 overexpression.

\section{Results}

Phenotypic characterization of mutant $\mathbf{3 7 9 0}$

Mutant 3790 was previously identified as an Arabidopsis thaliana activation tag mutant on which Myzus persicae shows a longer pre-reproductive period and produces smaller numbers of offspring than on its corresponding wild type Wassilewskija (Ws) [23]. Compared to Ws, mutant 3790 has smaller and darker green colored leaves (Figure 1), shows a delayed flowering, a reduced height of the main stem and an increased number of lateral branches.

\section{Identification of SKS13 as a gene conferring enhanced resistance to $M$. persicae}

Using inverse PCR we could determine that mutant 3790 contains a T-DNA including a $35 \mathrm{~S}$ enhancer that is located on chromosome 3 at position 4,350,852 (according to the TAIR website; http://www.arabidopsis.org) in the 3'-UTR region of the Brassinosteroid Receptor Like gene (BRL3, At3g13380; Figure 2a). Additionally, two other genes, SKU5 Similar 11 (SKS11, At3g13390) and SKU5 Similar 13 (SKS13, At3g13400) are located within a distance of approximately $8 \mathrm{~kb}$ of the enhancer (Figure 2a), a distance over which the enhancer can effectively activate the expression of genes [25]. To determine whether the transcript levels of these three genes were affected by the enhancer, we first performed quantitative RT-PCR (qPCR). The transcript level of BRL3 was two-fold higher in mutant 3790 than in Ws (Figure 2b). No transcripts of SKS11 and SKS13 were detectable in Ws but they could clearly be detected in mutant 3790 (Figure 2b).

As A. thaliana knockout mutants for many genes are publically available, we determined whether impaired expression of BRL3 affects the performance of $M$. persicae. To this purpose, we performed no-choice aphid assays and
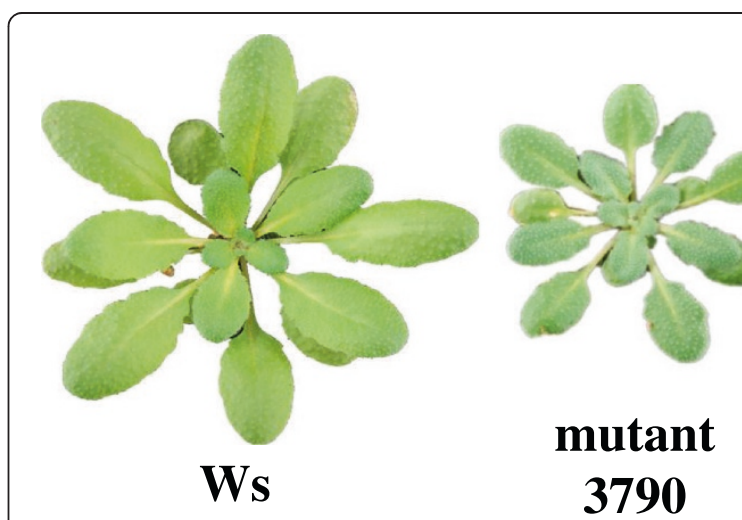

Figure 1 Phenotype of $\boldsymbol{A}$. thaliana mutant 3790. Rosette leaf phenotype of six-week old Wassilewskija (Ws) and mutant 3790. 


\section{a}

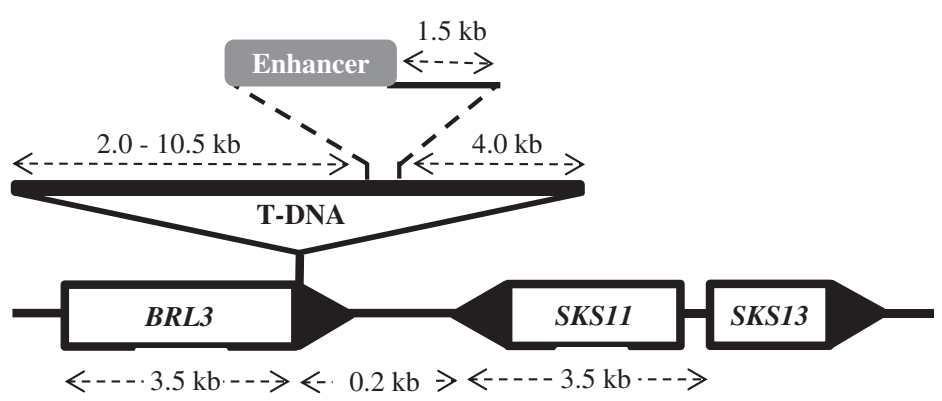

b

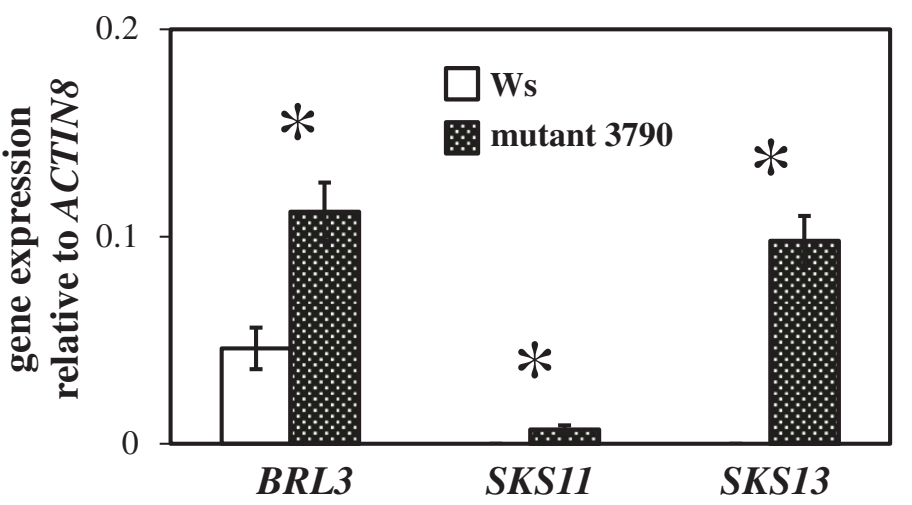

Figure 2 Location of the activation tag and expression analysis. (a) Genomic region of mutant 3790 showing the T-DNA insert containing the CaMV35S enhancer. The T-DNA is located in the 3'-UTR (black triangle) of BRL3. The exact distance between the adjacent genes SKS11 and SKS13 with their promoters and the enhancer is unknown. Diagram is not drawn to scale. (b) Quantitative RT-PCR expression analysis of BRL3, SKS11 and SKS13 in rosette leaves of Ws and mutant 3790. Values are the means \pm SD $(n=3)$. The star indicates a significant difference between bars within a pair (Independent-samples $t$-test, $P<0.05$ ).

compared M. persicae population development on BRL3 knockout mutants brl3-2 and brl3-3 [26] with that on wild type Columbia- 0 (Col-0). The numbers of $M$. persicae on these mutants $(18.5 \pm 5.6$ on brl3-2 and $16.2 \pm 4.1$ on brl3-3) did not differ from that on Col-0 (19.5 \pm 7.0 ; Kruskal-Wallis followed by Mann-Whitney $U$ test, $P>0.05$, $\mathrm{n}=15)$. Because SKS11 and SKS13 are not expressed in control leaves of Ws plants (Figure 2b), we performed a qPCR experiment to reveal whether these genes are induced upon infestation by $M$. persicae. Induced expression of Lipoxygenase 2 (LOX2; data not shown) indicated an efficient infestation of $M$. persicae [27], but the expression of SKS11 and SKS13 remained undetectable in Ws leaves six and 24 hours after infestation of $M$. persicae. Therefore, we did not evaluate $M$. persicae performance on SKS11 or SKS13 knockout mutants.

Due to the orientation regarding the position of the transposon (Figure 2a) and strongest overexpression impact (Figure 2b), we decided to focus on SKS13 for the continuation of this study. To confirm that overexpression of SKS13 enhances resistance to M. persicae, we generated transgenic Col-0 lines (G101, G102 and G103) in which SKS13 is overexpressed by the CaMV 35 S promoter. Compared to Col-0, these lines showed significantly higher expression levels of SKS13 (Figure 3a) and lower numbers of $M$. persicae (Figure $3 \mathrm{~b}$ ). Similar to mutant 3790 , plants of these transgenic lines had smaller, rounder rosette leaves than their corresponding wild type (Figure 3c), and delayed flowering. The height of the main stem and the numbers of lateral branches of plants from these transgenic lines did not differ from Col-0.

\section{Feeding behavior of $M$. persicae on mutant 3790}

To reveal whether aphid feeding behavior was affected by overexpression of SKS13 we compared electrical penetration graph (EPG) [1] recordings of $M$. persicae on mutant 3790 and Ws plants. The EPG parameters relevant for our study are summarized in Table 1. No differences were observed for EPG parameters related to epidermal or xylem tissue. Also the total time of the pathway phase was similar on Ws and mutant 3790 (Table 1). Myzus persicae showed a significantly longer duration of the non-probing phase on mutant 3790 than on Ws (Table 1). Significant differences were also observed for pre-phloem and phloem phase-related activities. Compared to Ws, M. persicae on mutant 3790 needed double the amount of time to the first phloem 

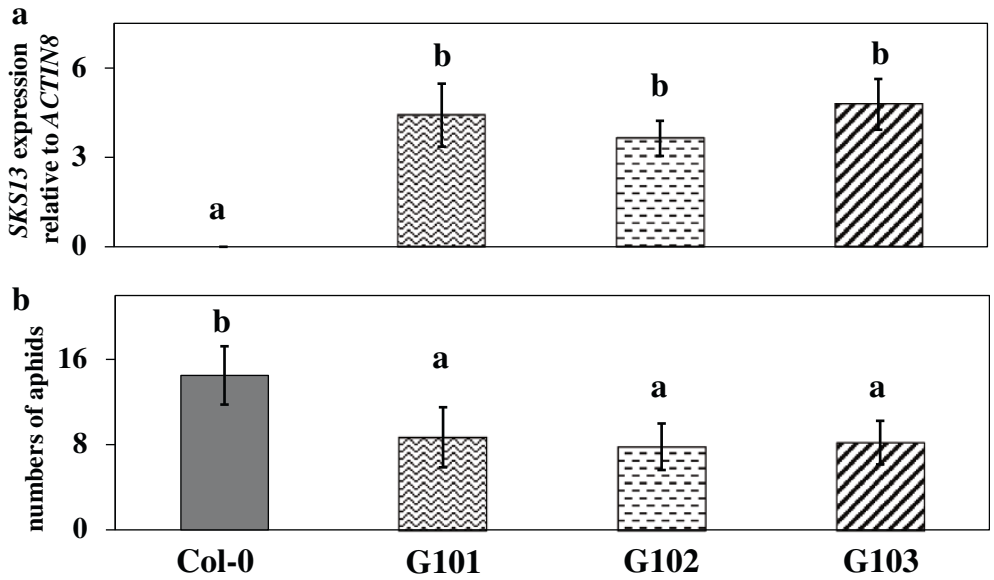

c

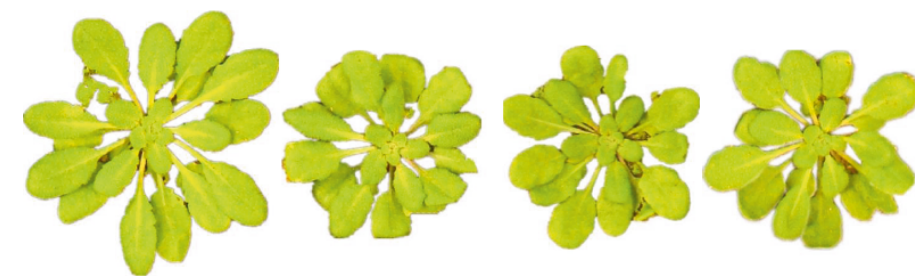

Figure 3 Gene expression analysis, Myzus persicae aphid performance and phenotype of three independent SKS13 overexpressing transgenic lines. (a) Quantitative RT-PCR expression analysis of SKS13 in rosette leaves of Columbia-0 (Col-0) and the three transgenic lines G101, G102 and G103. Values are the means \pm SD $(n=3)$. (b) Performance of $M$. persicae on plants of Col-0 and the three transgenic lines G101, G102 and G103. Values are the means $\pm S D(n=15)$. Bars marked with different letters are significantly different from each other (Kruskal-Wallis followed by Mann-Whitney $U$ tests, $P<0.05)$. (c) Rosette leaf phenotype of six-week-old Col-0 and transgenic lines G101, G102 and G103.

Table 1 Electrical Penetration Graph (EPG) parameters considered and their relation to Myzus persicae feeding activity on Arabidopsis thaliana Ws and mutant 3790

\begin{tabular}{|c|c|c|c|c|}
\hline Related tissue & EPG parameter & Wild type $(W s) n^{1}=18$ & Mutant $3790 \mathrm{n}=15$ & $P$ value ${ }^{2}$ \\
\hline Epidermal & Time to first probe (min) & $2.5 \pm 0.7$ & $2 \pm 0.4$ & 0.870 \\
\hline \multirow[t]{2}{*}{ Prephloem } & Number of probes before first phloem contact & $10.5 \pm 2.6$ & $13.2 \pm 4.6$ & 0.969 \\
\hline & Time from first probe to first phloem contact (min) & $61.6 \pm 12.4$ & $113.6 \pm 15.5$ & 0.024 \\
\hline \multirow[t]{9}{*}{ Phloem } & Total time of phloem salivation (min) & $10.8 \pm 1.5$ & $8.1 \pm 1.6$ & 0.025 \\
\hline & Number of phloem salivation events & $14.3 \pm 1.8$ & $10.1 \pm 1.8$ & 0.240 \\
\hline & Average duration of phloem salivation (min) & $0.8 \pm 0.1$ & $0.7 \pm 0.1$ & 0.462 \\
\hline & Total time of phloem ingestion (min) & $97.5 \pm 10.4$ & $33.3 \pm 8.2$ & 0.001 \\
\hline & Number of phloem ingestion events & $13.3 \pm 1.6$ & $8.7 \pm 1.7$ & 0.110 \\
\hline & Average duration of phloem ingestion (min) & $7.8 \pm 0.8$ & $4.3 \pm 1.0$ & 0.003 \\
\hline & Total time of sustained (>10 min) phloem ingestion & $64.2 \pm 9.4$ & $20.4 \pm 2.5$ & 0.002 \\
\hline & Number of sustained (>10 min) phloem ingestion & $3.9 \pm 0.6$ & $1.2 \pm 0.3$ & 0.001 \\
\hline & Average duration of sustained (>10 min) phloem ingestion & $17.4 \pm 2.5$ & $12.7 \pm 1.7$ & 0.032 \\
\hline \multirow[t]{3}{*}{ All tissues } & Total time of non-probing (min) & $106.4 \pm 16.9$ & $148.3 \pm 11.3$ & 0.023 \\
\hline & Total time of pathway phase (min) & $247.5 \pm 11.5$ & $261.4 \pm 13.3$ & 0.278 \\
\hline & Number of aphids with sustained (>10 min) phloem ingestion & $18.0(100 \%)$ & $11.0(73 \%)$ & 0.030 \\
\hline \multirow[t]{2}{*}{ Xylem } & Total time of $\mathrm{G}$ & $15.2 \pm 5.8$ & $16.2 \pm 4.7$ & 0.912 \\
\hline & Number of $\mathrm{G}$ & $0.7 \pm 0.3$ & $0.3 \pm 0.2$ & 0.195 \\
\hline
\end{tabular}

Values are means \pm SE of EPG parameters during $8 \mathrm{~h}$ monitoring. ${ }^{1}$ EPG replicates; ${ }^{2}$ Mann Whitney $\mathrm{U}$ (duration) or Fisher exact (number) test $P$ values. 
phase, but spend only about one third of the total time in this phase (Table 1). Additionally, fewer $M$. persicae showed sustained phloem sap ingestion on mutant 3790 than on WS and the ones that did show this activity on mutant 3790 did this a smaller number of times (Table 1). Furthermore, aphids on Ws spent significantly more time salivating into the phloem and ingesting phloem sap than aphids on mutant 3790 (Table 1).

Accumulation of reactive oxygen species in mutant line $\mathbf{3 7 9 0}$ SKS13 has a putative function in oxidation/reduction reactions [28,29] and its co-expressed genes function in the generation of reactive oxygen species (ROS) [30,31]. Therefore, we hypothesized that overexpression of SKS13 may lead to an accumulation of ROS in leaves. To visualize ROS we used 3-3'-diaminobenzidine (DAB) staining on the leaves of Ws, mutant 3790, Col-0 and transgenic line G101 (Figure 4). Each leaf was injured by forceps to serve as a positive control for the DAB staining [32]. In comparison to Ws and Col-0 leaves, darker browning was observed in leaves of mutant 3790 and transgenic line G010, respectively (Figure 4).

\section{Brevicoryne brassicae performance on mutant 3790}

It has been suggested that ROS accumulation plays a general role in plant defense against aphids $[11,12]$. Therefore, we hypothesized that SKS13 overexpressing plants would not only affect the generalist $M$. persicae but also other aphid species. This hypothesis was tested by infesting mutant 3790 and Ws with the specialist B. brassicae. At 14 days after infestation, an average of four B. brassicae was found on mutant 3790 and 18 B. brassicae on WS plants (Mann-Whitney $U$ test $P<0.001, \mathrm{n}=15$ ).

\section{Effect of SKS13 overexpression on transcription of known} JA-, SA- and ET-defense genes

To determine whether overexpression of SKS13 affects the plant hormone pathways known to be involved in plant defense against herbivorous insects, we monitored the expression levels of JA-, SA- and ET-marker genes in mutant 3790, Ws, SKS13 overexpressing transgenic lines and Col-0 without aphid infestation. In leaves of mutant 3790 the expression levels of the JA-marker genes LOX2 (Lipoxygenase 2), VSP2 (Vegetative Storage Protein 2) and PDF1.2 (Putative plant defensin 1.2) as well as SA-marker genes PAD4 (Phytoalexin Deficient4) and PR1 (Pathogenesis-related 1) were similar as in leaves of Ws (data not shown). However, the expression level of the ET-marker gene ERF1 (Ethylene response factor 1) was significantly higher in mutant 3790 than in WS (Figure 5). Conversely to mutant 3790 (in Ws background), the SKS13 overexpressing transgenic lines showed significant higher expression levels of the JA-marker genes compared to their corresponding wild type Col-0 (Figure 5). The SA- and ET-marker genes were not affected in these lines (data not shown).

\section{Discussion}

Overexpression of SKS13 in leaves enhances resistance to M. persicae in A. thaliana

Mutant 3790 was previously identified as an A. thaliana mutant on which the population development of $M$. persicae was reduced [23] and in the present paper we show that this is, at least partly, due to the constitutive overexpression of SKS13. The negative effect of SKS13 on aphid population development was confirmed in transgenic plants that embraced the SKS13 under the control of CaMV 35S promoter. An analysis of expression profiles in publicly available microarray data sets revealed that SKS13 is exclusively expressed in pollen (https://www.genev estigator.com/) [30]. This is in agreement with our observation that SKS13 was not expressed in leaves of Ws or Col-0. We also demonstrated that the expression of SKS13 was not induced upon infestation of $M$. persicae. This is consistent with previous microarray studies in which no induction of SKS13 expression in A. thaliana after $M$. persicae infestation was found [33-35].

\section{Overexpression of SKS13 affects feeding behavior of $M$. persicae probably due to ROS accumulation}

Analysis of $M$. persicae feeding behavior by the EPG technique can provide insight into the plant resistance

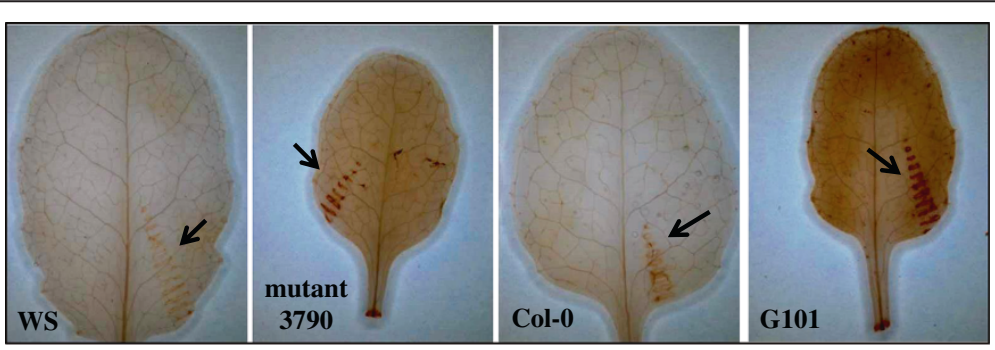

Figure 4 Accumulation of reactive oxygen species (ROS) in SKS13 overexpressing plants. 3-3'-diaminobenzidine (DAB) staining of detached leaves from Ws, mutant 3790, Col-0 and SKS13 overexpressing transgenic line G101. The arrows indicate the part of each leaf that was injured by forceps to serve as a positive control for the DAB staining. 

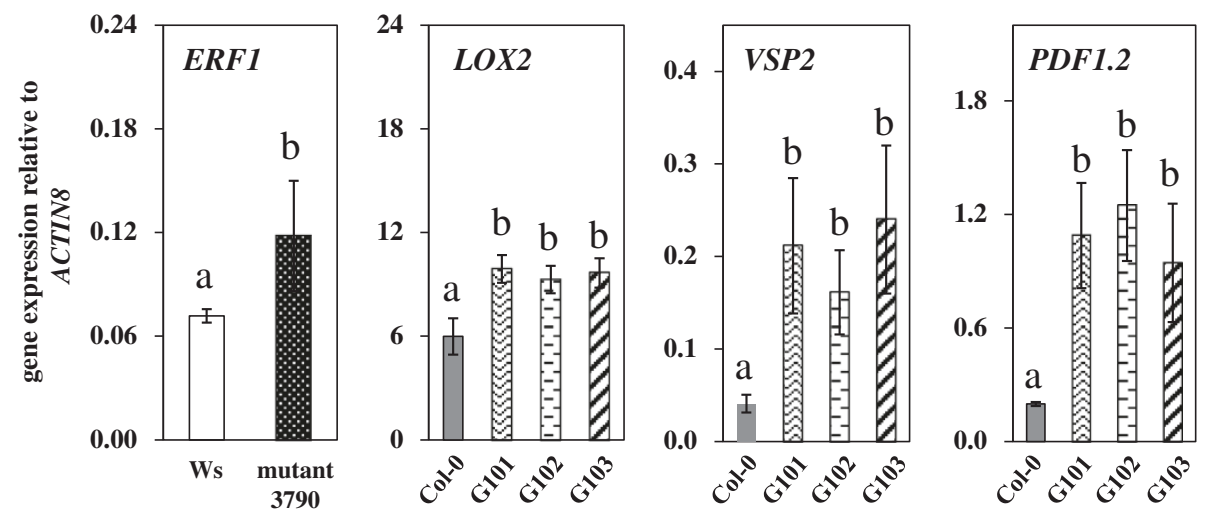

Figure 5 Expression analysis of ET and JA pathway marker genes in plants without aphid infestation. Quantitative RT-PCR data are shown for an ET marker gene (ERF1) in rosette leaves of Ws and mutant 3790, and for three JA marker genes (LOX2, VSP2 and PDF1.2) in rosette leaves of Col-0 and SKS13 overexpressing transgenic lines G101, G102 and G103. Values are the means \pm SD $(n=3)$. Bars marked with different letters are significantly different from each other within a graph (ANOVA followed by Tukey tests, $P<0.05$ ).

mechanisms [36]. The EPG results suggest that plant resistance conferred by overexpression of SKS13 was phloem based. This was supported by the fact that the phloem phase of M. persicae on SKS13 overexpressing plants was delayed in time and reduced in length, while the length of the pathway phase was not significantly different from the control. The phloem based resistance was further indicated by the reduced number of sustained phloem sap ingestions. As sustained phloem sap ingestion is required for the transmission of persistently transmitted viruses [37], the phloem based resistance explains the decreased transmission of such a virus, i.e. Turnip yellows virus, as previously observed in mutant 3790 [23].

To uncover the role of SKS13 in the phloem based plant resistance to $M$. persicae, we explored the possible biological function of this gene. As structurally related to multiple-copper oxidases, ascorbate oxidases and laccases, SKS13 has been suggested to function in oxidation/reduction reactions $[28,29]$. Furthermore, SKS13 is co-expressed with genes involved in ROS generation (https://www.genevestigator.com) [30,31]. Therefore we hypothesized that constitutive overexpression of SKS13 results in an accumulation of ROS in leaves and confirmed this by DAB staining the leaves of SKS13 overexpressing plants. The effect of ROS accumulation on aphid feeding behavior has also been shown for a triticale cultivar with a high concentration of ROS on which cereal aphids displayed a reduced time in the phloem phase and a prolonged time in the non-probe phase [38]. This is similar to our observations of $M$. persicae feeding behavior on SKS13 overexpressing plants. The accumulation of ROS was suggested to play a role in plant resistance to several aphid species [11,38]. This is also in line with our results, as aphid resistance on SKS13 overexpressing plants not only affected $M$. persicae but also $B$. brassicae performance.
Besides enhancing aphid resistance, excessive ROS can damage proteins, lipids and nucleic acids and can eventual be harmful to plant growth [39], thereby explaining the reduced size of SKS13 overexpressing plants.

\section{Overexpression of SKS13 affects plant hormone pathways}

\section{in A. thaliana}

Several studies suggest that ROS accumulation is linked with the JA, SA and ET plant hormone pathways to play a role in plant defense against aphids $[11,12,40,41]$. For instance, the $A$. thaliana RbohD mutant, in which JAinduced ROS accumulation does not occur, promotes a four times larger aphid population development than its wild type Col-0 [12,42], suggesting that aphid resistance conferred by activation of the JA pathway is probably mediated by ROS accumulation. In our study, we observed a similar activation of the JA pathway in SKS13 overexpressing Col-0 plants, as indicated by the significantly higher expression levels of three JA marker genes.

In mutant 3790, SKS13 is overexpressed in A. thaliana accession Ws and the ET pathway is activated instead of the JA pathway, which may be due to the genetic differences between Col-0 and Ws in response to ROS accumulation [43]. Furthermore, ROS may indirectly affect plant growth through altered signaling pathways. Kerchev et al. [44] concluded that a reduced Arabidopsis plant growth results from a low ascorbate, a compound that buffers the production of ROS, that triggers ABA- and JA dependent signaling. As ascorbate buffers the production of ROS, low levels of this compound would result in enhanced ROS accumulation. This is consistent with the observed higher ROS accumulation and reduced plant growth for both mutant 3790 and SKS13 overexpressing transgenic lines in our study. Our observation that signaling pathways were differently affected suggests that other factors may influence plant growth as well. In addition to SKS13, 
the higher expression of BRL3 and/or SKS11 may contribute to this difference. Alternatively the additional differences may be attributed to unknown interactions among BR, ET and ROS. Studying the interaction between SKS13 and JA-/ET-mediated defense responses may lead to a better understanding of activation of JA and ET responses and their contribution to aphid resistance.

\section{Conclusions}

Overexpression of SKS13 in A. thaliana leads to a reduced phloem feeding of $M$. persicae, which probably is due to accumulation of ROS in leaves. The reduced phloem feeding results in the suppression of the population development of $M$. persicae and also decreases the transmission of persistent viruses. Overexpression of SKS13 in A. thaliana also affects $B$. brassicae and possibly other phloem feeding insects as well. The enhanced resistance towards $M$. persicae and B. brassicae in SKS13 overexpressing A. thaliana plants reduces plant development.

\section{Methods \\ Plants}

Mutant 3790, was previously identified from an Arabidopsis thaliana accession Wassilewskija (Ws) activation tag library as a mutant on which $M$. persicae showed a reduced population development [23]. Seeds of this mutant and its corresponding background accession Ws were obtained from the library present at Wageningen UR Plant Breeding [22]. Seeds of brl3-2 and brl3-3 mutants and their corresponding background accession Columbia-0 (Col-0) were kindly provided by Prof. S.C. de Vries, Laboratory of Biochemistry Wageningen University [26]. To induce germination, seeds were placed at $4^{\circ} \mathrm{C}$ in the dark for 3 days under high humidity. Subsequently, seeds were transferred to potting compost (Lentse Potgrond ${ }^{\oplus}$ ) and plants were cultivated in a climate chamber $\left(20 \pm 2{ }^{\circ} \mathrm{C}\right.$, RH 60-70\%, 6 h: 18 h (light: dark). Plants were watered every other day and no pest control was applied. In all experiments we used plants in their vegetative stage, i.e. before they start flowering.

\section{Insects}

Myzus persicae (green peach aphid) was reared in cages on Chinese cabbage (Brassica rapa L. ssp. pekinensis cv. Granaat). Brevicoryne brassicae (cabbage aphid) was reared on Brussels sprouts (Brassica oleracea L. var. gemmifera cv. Cyrus) at the Laboratory of Entomology, Wageningen University. Both rearings were maintained in an acclimatized room at $20 \pm 2^{\circ} \mathrm{C}$, RH 60-70\%, 18 h: 6 h (light: dark). For all experiments, only apterous aphids were used.

\section{Inverse PCR}

Genomic DNA was extracted from leaves of mutant 3790 using the DNeasy Plant Mini kit (Qiagen), digested with restriction enzyme EcoRI (Thermo, product \# ER0275) or BamHI (Thermo, product \# ER0051) and subsequently ligated with T4 DNA ligase (Fermentas, product \# EL0011). Inverse PCR was performed according to the method described previously [21]. PCR products were sequenced and then blasted against the $A$. thaliana genome (http://www.arabidopsis.org/) [45].

\section{Time course experiment of $M$. persicae infestation}

To determine if the SKS11 and SKS13 gene are induced upon $M$. persicae infestation, we performed a time course experiment. Four-week-old plants were infested with 15 wingless aphids of assorted life stages. Leaf material was collected after zero, six and 24 hours of aphid infestation. Aphids were gently brushed away from the leaf tissue and uninfested plants were also brushed. Leaf material was immediately flash frozen in liquid nitrogen and stored at $-80^{\circ} \mathrm{C}$ until use.

\section{Quantitative RT-PCR}

Samples were designed in three biological replicates, with 17 individual plants pooled per replicate. Total RNA isolation, cDNA synthesis and quantitative RT-PCR (qPCR) were performed according to the methods described previously [21]. Gene specific primers were designed with Primer-3-Plus software [46] and are listed in Table 2. Threshold cycle (Ct) values obtained with qPCR were normalized for differences in cDNA synthesis by subtracting the Ct value of the constitutively expressed gene ACTIN8 (At1g49240) [47] from the $\mathrm{Ct}$ value of the gene of interest. Normalized gene expression was then calculated as $2^{-\Delta C T}$ and Log-transformed prior to analysis. Independent-samples $t$-test or ANOVA followed by Tukey tests were used to determine the significance between genotypes/treatments $(P<0.05)$.

\section{Generation of transgenic plants}

To generate transgenic lines overexpressing SKS13, the coding region fragment of SKS13 was amplified from

\section{Table 2 Sequences of gene specific primers used for} quantitative RT-PCR analyses

\begin{tabular}{|c|c|c|}
\hline Gene name & Forward primer $\left(5^{\prime} \rightarrow 3^{\prime}\right)$ & Reverse primer $\left(5^{\prime} \rightarrow 3^{\prime}\right)$ \\
\hline$B R L 3$ & GGACATACCCGGGAGTACCT & CCCGTGTCTCAGATTITGGT \\
\hline SKS11 & CAACTGTGGAATGTGGAACG & GGTGACAAGACACTCGCGTA \\
\hline SKS13 & GAGCTACGAAGGAAGCAACG & CACTGGCGGTTAAGTTCCAT \\
\hline LOX2 & AGATTCAAAGGCAAGCTCCA & ACAACACCAGCTCCAGCTCT \\
\hline VSP2 & TACGAACGAAGCCGAACTCT & GGCACCGTGTCGAAGTCTAT \\
\hline PDF1.2 & САCСCTTATCTTCGCTGCTC & GCACAACTTCTGTGCTTCCA \\
\hline PAD4 & GTTCTITTCCCCGGCTTATC & CGGTTATCACCACCAGCTTT \\
\hline PR1 & GGCCTTACGGGGAAAACTTA & CTCGCTAACCCACATGTTCA \\
\hline ERF1 & CTTCCGACGAAGATCGTAGC & TCTTGACCGGAACAGAATCC \\
\hline ACTIN8 & GATGGAGACCTCGAAAACCA & AAAAGGACTTCTGGGCACCT \\
\hline
\end{tabular}


Col-0 cDNA using primers AttB1_SKS13_F (GGGGA CAAGTTTGTACAAAAAAGCAGGCTCGAGCGAGA GAGATTCAAAAAT) and AttB2_SKS13_R (GGGGAC CACTTTGTACAAGAAAGCTGGGTTCCTCTC TGG ATTGAACAATGA) in a PCR reaction containing the Phusion $^{\text {th }}$ enzyme (Finnzymes, Product codes: F-530S, $100 \mathrm{U})$. The following PCR program was used: $30 \mathrm{sec}-$ onds at $98^{\circ} \mathrm{C}$ followed by 35 cycles of $98^{\circ} \mathrm{C}$ for $10 \mathrm{sec}$, $64^{\circ} \mathrm{C}$ for $10 \mathrm{sec}$, and $72^{\circ} \mathrm{C}$ for 3 min with a final extension at $72^{\circ} \mathrm{C}$ for $10 \mathrm{~min}$. The resulting PCR product was extracted from a $1 \%$ agarose gel using the QIAquick Gel Extraction Kit (Qiagen) and sequenced for verification. The verified coding region fragment of SKS13 was transferred into donor vector pDONR207 using the Gateway $^{\circ}$ BP Clonase ${ }^{\mathrm{Tu}}$ II enzyme mix (Invitrogen) to generate entry vector pDONR207::SKS13. The entry vector was subsequently cloned into Gateway destination vector pFAST-R02 [48] using the Gateway LR ${ }^{\circ}$ Clonase $^{\text {rux }}$ II enzyme mix (Invitrogen) to generate the expression construct pFAST-R02-SKS13 in which SKS13 is under the control of the CaMV 35S promoter. The construct was transformed into E. coli and transformants were checked by colony PCR using primers AttB1_F (GGGGACAAGTT TGTACAAAAAAGCAGGCT) and AttB2_R (ACCACTT TGTACAAGAAAGCTG GGT). After verifying the accuracy of the coding region fragment of SKS13, the construct was transformed into Agrobacterium tumefaciens strain GV3101 [49] by electroporation. Agrobacterium mediated transformation [50] was used to introduce the pFASTR02-SKS13 plasmid into Col-0 flowers. Seeds containing the construct were selected using fluorescence microscopy (Zeiss, SteREO Discovery.V8) [48].

\section{No-choice aphid assays}

Nymph producing adult aphids (both $M$. persicae and $B$. brassicae) were collected from rearing plants and placed on detached cabbage leaves in a petri dish overnight. New born one-day-old nymphs were placed in the centre of three-week-old $A$. thaliana plants using a fine brush. Each plant received one nymph and the total number of aphids was counted 14 days after infestation. The plants were randomly organized with 15 biological replicates per genotype. Plants were separated by a water barrier to prevent aphids crossing over from one plant to the other. The Mann-Whitney $U$ test or Kruskal-Wallis followed by Mann-Whitney $U$ test were used to determine if differences between genotypes were significant $(P<0.05)$.

\section{Electrical penetration graph}

The electrical penetration graph (EPG) technique [24] was employed to monitor the feeding behavior of Myzus persicae. A gold wire (diameter $20 \mu \mathrm{m}$ ) was attached onto the dorsum of young adult aphids using conductive water-based silver glue. The wired aphid was placed on a mature leaf of a five-week-old plant that was connected to a recording system via a copper electrode in the soil. All tested aphids stayed at the underside of the leaf. The EPGs were recorded at $22^{\circ} \mathrm{C}$ with constant light for 8 hours. The EPG data were analyzed using the PROBE 3.0 software (Wageningen University, the Netherlands) to distinguish the various waveforms. Waveform $\mathrm{C}$ represents the pathway phase, when the aphid stylet is penetrating through the leaf tissue; waveform E2 represents phloem sap ingestion; Waveform $\mathrm{F}$ is associated with penetration difficulties; and waveform $G$ indicates active uptake of water from the xylem. Both sequential and non-sequential parameters were analyzed [51] to characterize probing behavior of individual $M$. persicae. At least 15 recordings of individual aphids (one aphid per plant) were obtained for each genotype. The Mann-Whitney $U$ and Fisher exact test were used to determine the significance difference between genotypes $(P<0.05)$.

\section{Determination of reactive oxygen species (ROS) accumulation}

To visualize reactive oxygen species (ROS), leaves were cut from four-week-old plants and submerged overnight in an $\mathrm{HCl}$ solution containing $1 \mathrm{mg} \mathrm{mL} \mathrm{m}^{-1} 3$-3'-diaminobenzidine (DAB), pH 3.7 [52]. Chlorophyll was extracted with $96 \%$ ethanol overnight at room temperature. Leaves were subsequently photographed in $80 \%$ glycerol.

\section{Competing interests}

The authors declare that they have no competing interests.

\section{Authors' contributions}

XC carried out the molecular and insect behavior studies, performed the DAB staining study, analyzed the data and drafted the manuscript. ZZ generated the transgenic overexpressing lines. RV helped to draft the manuscript. BV and CB conceived of the study, participated in its design and coordination and helped to draft the manuscript. All authors read and approved the final manuscript.

\section{Acknowledgements}

The authors thank Cindy ten Broeke and Freddy Tjallingii for assistance with the EPG recordings; Gerrit Stunnenberg and Taede Stoker for taking care of the plants; Greet Steenhuis-Broers and Leon Westerd for looking after the aphid rearings; Roeland Voorrips for suggestions on statistics; Wei Liu for assistance on molecular analysis; Weicong Qi for valuable discussions; three anonymous reviewers for their valuable comments and suggestions.

\section{Author details}

'Wageningen UR, Plant Breeding, PO. Box 386, Wageningen 6700 AJ, the Netherlands. 'Laboratory of Phytopathology, Wageningen University, Droevendaalsesteeg 1, 6708PB, Wageningen, the Netherlands.

Received: 28 March 2014 Accepted: 4 August 2014

Published: 14 August 2014

\section{References}

1. Tjallingii WF: Salivary secretions by aphids interacting with proteins of phloem wound responses. J Exp Bot 2006, 57:739-745.

2. Will T, Kornemann SR, Furch AC, Tjallingii WF, van Bel AJ: Aphid watery saliva counteracts sieve-tube occlusion: a universal phenomenon? J Exp Biol 2009, 212:3305-3312. 
3. Moreno A, Garzo E, Fernandez-Mata G, Kassem M, Aranda MA, Fereres A: Aphids secrete watery saliva into plant tissues from the onset of stylet penetration. Entomol Exp App/ 2011, 139:145-153.

4. Brault V, Uzest M, Monsion B, Jacquot E, Blanc S: Aphids as transport devices for plant viruses. Comp Ren Biol 2010, 333:524-538.

5. Howe GA, Jander G: Plant immunity to insect herbivores. Annu Rev Plant Biol 2008, 59:41-66.

6. Alvarez AE, Tjallingii WF, Garzo E, Vleeshouwers V, Dicke M, Vosman B: Location of resistance factors in the leaves of potato and wild tuber-bearing Solanum species to the aphid Myzus persicae. Entomol Exp Appl 2006, 121:145-157.

7. Halkier BA, Gershenzon J: Biology and biochemistry of glucosinolates. Annu Rev Plant Biol 2006, 57:303-33.

8. Kim JH, Lee BW, Schroeder FC, Jander G: Identification of indole glucosinolate breakdown products with antifeedant effects on Myzus persicae (green peach aphid). Plant J 2008, 54:1015-1026.

9. Maffei ME, Mithöfer A, Boland W: Before gene expression: early events in plant-insect interaction. Trends Plant Sci 2007, 12:310-316.

10. Kerchev PI, Fenton B, Foyer $\mathrm{CH}$, Hancock RD: Plant responses to insect herbivory: interactions between photosynthesis, reactive oxygen species and hormonal signalling pathways. Plant Cell Environ 2012, 35:441-453.

11. Moloi MJ, van der Westhuizen AJ: The reactive oxygen species are involved in resistance responses of wheat to the Russian wheat aphid. J Plant Physiol 2006, 163:1118-1125

12. Miller G, Schlauch K, Tam R, Cortes D, Torres MA, Shulaev V, Dangl JL, Mittler R: The plant NADPH oxidase RBOHD mediates rapid systemic signaling in response to diverse stimuli. Sci Signal 2009, 2:ra45.

13. Wu J, Baldwin IT: New insights into plant responses to the attack from insect herbivores. Annu Rev Genet 2010, 44:1-24.

14. Morkunas I, Mai V, Gabrys B: Phytohormonal signaling in plant responses to aphid feeding. Acta Physiol (Oxf) 2011, 33:2057-2073.

15. Robert-Seilaniantz A, Grant M, Jones JDG: Hormone crosstalk in plant disease and defense: More than just JASMONATE-SALICYLATE antagonism. Annu Rev Plant Biol 2011, 49:317-343.

16. Thompson GA, Goggin FL: Transcriptomics and functional genomics of plant defence induction by phloem-feeding insects. J Exp Bot 2006 57:755-766

17. Zhu-Salzman K, Bi J-L, Liu T-X: Molecular strategies of plant defense and insect counter-defense. Insect Sci 2005, 12:3-15.

18. Levy M, Wang Q, Kaspi R, Parrella MP, Abel S: Arabidopsis IQD1, a novel calmodulin-binding nuclear protein, stimulates glucosinolate accumulation and plant defense. Plant J 2005, 43:79-96.

19. Zhang Z, Ober JA, Kliebenstein DJ: The gene controlling the quantitative trait locus EPITHIOSPECIFIER MODIFIER1 alters glucosinolate hydrolysis and insect resistance in Arabidopsis. Plant Cell 2006, 18:1524-1536.

20. Louis J, Lorenc-Kukula K, Singh V, Reese J, Jander G, Shah J: Antibiosis against the green peach aphid requires the Arabidopsis thaliana MYZUS PERSICAE-INDUCED LIPASE1 gene. Plant J 2010, 64:800-811.

21. Chen X, Zhang Z, Visser RGF, Broekgaarden C, Vosman B: Overexpression of IRM1 enhanced resistance to aphids in Arabidopsis thaliana. PLoS One 2013, 8:e70914.

22. Marsch-Martinez N, Greco R, Van Arkel G, Herrera-Estrella L, Pereira A: Activation tagging using the En-I maize transposon system in Arabidopsis. Plant Physiol 2002, 129:1544-1556.

23. Chen X, Vosman B, Visser RGF, van der Vlugt RA, Broekgaarden C: High throughput phenotyping for aphid resistance in large plant collections. Plant Methods 2012, 8:33-33.

24. Tjallingii WF: Continuous recording of stylet penetration activities by aphids. In Aphid-plant genotype interactions. Edited by Campbell RK, Eikenbary RD. Amsterdam, NL: Elsevier Science Publicing; 1990:89-99.

25. Ichikawa T, Nakazawa M, Kawashima M, Muto S, Gohda K, Suzuki K, Ishikawa A, Kobayashi H, Yoshizumi T, Tsumoto Y, Tsuhara Y, lizumi H, Goto Y, Matsui M: Sequence database of 1172 T-DNA insertion sites in Arabidopsis activation-tagging lines that showed phenotypes in T1 generation. Plant J 2003, 36:421-429.

26. Caño-Delgado A, Yin Y, Yu C, Vafeados D, Mora-García S, Cheng J-C, Nam KH, Li J, Chory J: BRL1 and BRL3 are novel brassinosteroid receptors that function in vascular differentiation in Arabidopsis. Development 2004, 131:5341-5351.

27. Moran PJ, Thompson GA: Molecular responses to aphid feeding in Arabidopsis in relation to plant defense pathways. Plant Physiol 2001 125:1074-1085
28. Sedbrook JC, Carroll KL, Hung KF, Masson PH, Somerville CR: The Arabidopsis SKU5 gene encodes an extracellular glycosyl phosphatidylinositol-anchored glycoprotein involved in directional root growth. Plant Cell 2002, 14:1635-1648.

29. Lamesch P, Berardini TZ, Li D, Swarbreck D, Wilks C, Sasidharan R, Muller R, Dreher K, Alexander DL, Garcia-Hernandez M, Karthikeyan AS, Lee CH, Nelson WD, Ploetz L, Singh S, Wensel A, Huala E: The Arabidopsis Information Resource (TAIR): improved gene annotation and new tools. Nucleic Acids Res 2012, 40:D1202-D1210.

30. Hruz T, Laule O, Szabo G, Wessendorp F, Bleuler S, Oertle L, Widmayer P, Gruissem W, Zimmermann P: Genevestigator V3: A reference expression database for the meta-analysis of transcriptomes. Adv Bioinformatics 2008, Article ID 420747.

31. Wang C-L, Wu J, Xu G-H, Gao Y-B, Chen G, Wu J-Y, Wu H-Q, Zhang S-L: S-RNase disrupts tip-localized reactive oxygen species and induces nuclear DNA degradation in incompatible pollen tubes of Pyrus pyrifolia. J Cell Sci 2010, 123:4301-4309.

32. Takahashi F, Mizoguchi T, Yoshida R, Ichimura K, Shinozaki K. Calmodulin-dependent activation of MAP Kinase for ROS homeostasis in Arabidopsis. Mol Cell 2011, 41:649-660.

33. Moran PJ, Cheng Y, Cassell JL, Thompson GA: Gene expression profiling of Arabidopsis thaliana in compatible plant-aphid interactions. Arch Insect Biochem Physiol 2002, 51:182-203.

34. De Vos M, Van Oosten VR, Van Poecke RM, Van Pelt JA, Pozo MJ, Mueller MJ, Buchala AJ, Metraux JP, Van Loon LC, Dicke M, Pieterse CMJ: Signal signature and transcriptome changes of Arabidopsis during pathogen and insect attack. Mol Plant Microbe Interact 2005, 18:923-937.

35. Kempema LA, Cui X, Holzer FM, Walling LL: Arabidopsis transcriptome changes in response to phloem-feeding silverleaf whitefly nymphs. Similarities and distinctions in responses to aphids. Plant Physiol 2007, 143:849-865.

36. Tjallingii WF, Garzo E, Fereres A: New structure in cell puncture activities by aphid stylets: a dual-mode EPG study. Entomol Exp App/ 2010, 135:193-207.

37. Stafford CA, Walker GP, Ullman DE: Hitching a ride: Vector feeding and virus transmission. Commun Integr Biol 2012, 5:43-49.

38. Łukasik I, Goławska S, Wójcicka A: Effect of cereal aphid infestation on ascorbate content and ascorbate peroxidase activity in triticale. Polish $J$ Environ Stud 2012, 21:1937-1941.

39. Apel K, Hirt H: Reactive oxygen species: Metabolism, oxidative stress, and signal transduction. Annu Rev Plant Physiol Plant Mol Biol 2004, 55:373-399.

40. Argandoña VH, Chaman M, Cardemil L, Muñoz O, Zúñiga GE, Corcuera LJ: Ethylene production and peroxidase activity in aphid-infested barley. J Chem Ecol 2001, 27:53-68.

41. Mohase $L$, van der Westhuizen AJ: Salicylic acid is involved in resistance responses in the Russian wheat aphid-wheat interaction. J Plant Physiol 2002, 159:585-590.

42. Maruta T, Inoue T, Tamoi M, Yabuta Y, Yoshimura K, Ishikawa T, Shigeoka S Arabidopsis NADPH oxidases, AtrbohD and AtrbohF, are essential for jasmonic acid-induced expression of genes regulated by MYC2 transcription factor. Plant Sci 2011, 180:655-660.

43. Ahmad S, Van Hulten M, Martin J, Pieterse CMJ, Van Wees SCM, Ton J: Genetic dissection of basal defence responsiveness in accessions of Arabidopsis thaliana. Plant Cell Environ 2011, 34:1191-1206.

44. Kerchev PI, Pellny TK, Vivancos PD, Kiddle G, Hedden P, Driscoll S: The transcription factor $A B \mid 4$ Is required for the ascorbic acid-dependent regulation of growth and regulation of jasmonate-dependent defense signaling pathways in Arabidopsis. Plant Cell 2011, 23:3319-3334.

45. Altschul SF, Gish W, Miller W, Myers EW, Lipman DJ: Basic local alignment search tool. J Mol Biol 1990, 215:403-410.

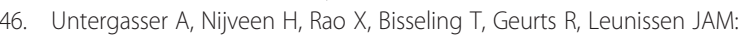
Primer3Plus, an enhanced web interface to Primer3. Nucleic Acid Res 2007, 35:W71-W74.

47. Iven T, König S, Singh S, Braus-Stromeyer SA, Bischoff M, Tietze LF, Braus GH, Lipka V, Feussner I, Dröge-Laser W: Transcriptional activation and production of tryptophan-derived secondary metabolites in Arabidopsis roots contributes to the defense against the fungal vascular pathogen Verticillium longisporum. Mol Plant 2012, 5:1389-1402.

48. Shimada TL, Shimada T, Hara-Nishimura I: A rapid and non-destructive screenable marker, FAST, for identifying transformed seeds of Arabidopsis thaliana. Plant J 2010, 61:519-528. 
49. Hellens RP, Edwards EA, Leyland NR, Bean S, Mullineaux PM: pGreen: a versatile and flexible binary $\mathrm{Ti}$ vector for Agrobacterium-mediated plant transformation. Plant Mol Biol 2000, 42:819-832.

50. Clough SJ, Bent AF: Floral dip: a simplified method for Agrobacterium-mediated transformation of Arabidopsis thaliana. Plant J 1998, 16:735-743.

51. Sarria E, Cid M, Garzo E, Fereres A: Excel Workbook for automatic parameter calculation of EPG data. Comp Elec Agricult 2009, 67:35-42.

52. Orozco-Cardenas M, Ryan CA: Hydrogen peroxide is generated systemically in plant leaves by wounding and systemin via the octadecanoid pathway. Proc Natl Acad Sci U S A 1999, 96:6553-6557.

doi:10.1186/s12870-014-0217-3

Cite this article as: Chen et al: Constitutive overexpression of the pollen specific gene SKS13 in leaves reduces aphid performance on Arabidopsis thaliana. BMC Plant Biology 2014 14:217.

\section{Submit your next manuscript to BioMed Central and take full advantage of:}

- Convenient online submission

- Thorough peer review

- No space constraints or color figure charges

- Immediate publication on acceptance

- Inclusion in PubMed, CAS, Scopus and Google Scholar

- Research which is freely available for redistribution 